\title{
Surveillance
}

\section{HIV in Canada: 2009 to 2014}

\author{
Tomas $\mathrm{K}^{1 *}$, Dhami $\mathrm{P}^{1}$, Houston $\mathrm{C}^{1}$, Ogunnaike-Cooke $\mathrm{S}^{1}$, Rank $\mathrm{C}^{1}$ \\ ${ }^{1}$ Public Health Agency of Canada, Ottawa, ON \\ *Correspondence: kristina.tomas@phac-aspc.gc.ca
}

\begin{abstract}
Background: Between 1996 and 2008, the number of newly reported HIV cases in Canada fluctuated between approximately 2,100 and 2,700 cases per year.
\end{abstract}

Objective: To describe the recent trends in new diagnoses of HIV in Canada between 2009 and 2014 by age group, sex, exposure category, race/ethnicity, and region, as well as the number of perinatally HIV-exposed infants.

Methods: HIV data were compiled from two databases. The National HIVIAIDS Surveillance System is a passive surveillance system that gathers non-nominal data on a voluntary basis from all the provinces and territories of all cases that meet the national case definition, and includes data on age group, sex, race/ethnicity, country of birth, and exposure categories. The Canadian Perinatal HIV Surveillance Program is a sentinel-based surveillance system; non-nominal data is obtained through a national confidential survey completed by participating physicians.

Results: Since 2009 the number of new HIV cases has slowly but steadily declined from 2,391 cases in 2009 to 2,044 in 2014, which is the lowest number of annual HIV cases seen in the last two decades. The largest proportion (32\%) of new HIV cases continues to be diagnosed among those 30 to 39 years of age, but the proportion of cases diagnosed among those 50 years of age or over has increased from $15 \%$ in 2009 to slightly over $20 \%$ in 2014 . Approximately $75 \%$ of newly diagnosed cases are males and $25 \%$ females. In males, the most common exposure category $(60 \%)$ was men who have sex with men. In females, the most common exposure category $(66 \%)$ was heterosexual contact followed by injection drug use $(27 \%)$. Race/ethnicity varied by sex. In males, over $50 \%$ were White, $14 \%$ were Aboriginal and $13 \%$ Black. In females, $35 \%$ were Black, $35 \%$ were Aboriginal and $22 \%$ were White. There were regional variations in HIV rates across Canada. Between 2009 and 2014, the number of perinatally HIV-exposed infants varied between 200 and 249 but the percentage of perinatal treatment increased from $87 \%$ in 2009 to $97 \%$ in 2014 .

Conclusion: The annual number of reported HIV cases in Canada has been declining in recent years. The proportion of HIV-positive mothers receiving treatment has increased and the number of confirmed HIV-infected infants has decreased.

\section{Introduction}

According to the World Health Organization, there are 36.9 million people worldwide living with HIV and 2.0 million people were newly infected with HIV in 2014 (1). HIV remains a public health concern both in Canada and internationally. One of the key programmatic responses in Canada and globally is the timely diagnosis of people with HIV, which benefits individuals with HIV (where early care and appropriate treatment can result in prolonged and improved quality of life) and the overall population (since people 
who know their HIV-positive status are more likely to adopt strategies to reduce the possibility of onward transmission of the virus). Timely diagnosis is a key focus of the newly released UNAIDS 90-90-90 targets which aim for $90 \%$ of all people living with HIV to know their HIV status by the year 2020 (2).

National reporting of HIV cases began in 1985. Between 1996 and 2008, the number of newly reported HIV cases in Canada fluctuated between approximately 2,100 to 2,700 cases per year; however, the number of people living with HIV was much higher. According to the Public Health Agency of Canada (the Agency), there were approximately 75,500 people living with HIV (including AIDS) in Canada at the end of 2014 (3). Since new HIV treatments have reduced HIV mortality and new infections continue to occur at a rate greater than the number of deaths among people living with HIV, the overall number of Canadians living with HIV infection will likely continue to increase in the years to come.

This article provides a brief overview of the epidemiology of newly reported cases of HIV in Canada between 2009 and 2014 and summarizes recent trends by geographic location, sex, age group, exposure category, and race/ethnicity. The data presented here are based on HIVIAIDS in Canada: Surveillance Report to December 31, 2014 (4). The HIVIAIDS Surveillance Report series is published annually and provides an overview of HIV and AIDS case surveillance data in Canada from a national perspective.

\section{Methods}

\section{Data collection}

HIV data are compiled at the national level in Canada using four data sources: the National HIVIAIDS Surveillance System (Public Health Agency of Canada); the Canadian Perinatal HIV Surveillance Program (Canadian Pediatric AIDS Research Group); immigration medical exam data from Citizenship and Immigration Canada and Vital Statistics data from Statistics Canada. For the purpose of this summary we will focus on the first two.

\section{National HIV/AIDS Surveillance System}

The National HIVIAIDS Surveillance System (HASS) is a passive case-based surveillance system that collates data voluntarily submitted to the Agency on an annual basis from all provincial and territorial public health authorities.

HIV is a nationally notifiable disease which means that this condition has been prioritized for national level monitoring. This system is voluntary, with cases reported through provincial or territorial departments of health. Provinces and territories have their own legislation for the reporting of priority infectious diseases within their jurisdictions; in 2015, all provinces and territories now include mandatory reporting of HIV (with some variation in specific case definition requirements).

Although HIV reporting requirements and practices differ across the country (5), cases reported to the Agency must meet the national case definition which requires laboratory evidence of HIV infection through a confirmed, repeatedly reactive screening test for HIV antibody in a person over 15 months of age or for cases with history of non-maternal-fetal HIV transmission. See textbox below for the national HIV case definition. 


\section{National HIV case definition (6)}

Adults, adolescents and children $\geq 18$ months of age:

Detection of HIV antibody with confirmation (e.g., enzyme immunoassay (EIA) screening with confirmation by Western blot or other confirmatory test) OR detection of HIV nucleic acid (e.g., DNA polymerase chain reaction (PCR) or plasma RNA) OR HIV p24 antigen with confirmation by neutralization assay OR isolation of HIV in culture Children <18 months of age (on two separate samples collected at different times):

Detection of HIV nucleic acid (e.g., DNA PCR or plasma RNA) OR HIV p24 antigen with confirmation by neutralization assay OR isolation of HIV in culture

The HIV surveillance database consists of non-nominal data on people diagnosed with HIV infection including, but not limited to age group, sex, race/ethnicity, country of birth, and risks associated with the transmission of HIV that are organized into exposure categories (see textbox on exposure categories below).

\section{Determination of exposure category for HIV}

HIV and AIDS cases are assigned to a single exposure category according to a hierarchy of risk factors. If more than one risk factor is reported, a case is classified according to the exposure category listed first (or highest) in the hierarchy. For example, people who inject drugs may also be at risk of HIV infection through heterosexual sexual activity. However, injection drug use (IDU) is accepted as the higher risk activity with greater likelihood of transmission of HIV. The hierarchy is as follows:

- Perinatal transmission

- MSM-IDU (men who have sex with men and inject drugs)

- $\operatorname{MSM}$ (men who have sex with men)

- IDU (injection drug use)

- Recipient of blood/blood products

- Heterosexual contact (origin from an HIV-endemic country; sexual contact with a person at risk; or no identified risk/heterosexual)

- Occupational exposure

- Other

- No identified risk

Standardized data recoding procedures were applied to all submitted provincial and territorial datasets to create a national dataset for analysis.

\section{Canadian Perinatal HIV Surveillance Program}

National data on the HIV status of infants exposed perinatally to HIV infection are collected through the Canadian Perinatal HIV Surveillance Program (CPHSP), an initiative of the Canadian Pediatric AIDS Research Group (CPARG). Support for the CPHSP is provided by the Canadian HIV Trials Network (CTN) and the Surveillance and Epidemiology Division within the Agency.

The CPHSP is a sentinel-based surveillance system that collects data on all identified infants and children in Canada born to mothers who are known to be infected with HIV (7). The CPHSP includes infants identified as exposed to HIV during pregnancy, older infants and children not identified in the perinatal period, and those born outside Canada who are receiving care for HIV infection. Data were obtained through a national, non-nominal, confidential survey of infants known to participating pediatricians in tertiary care centres and specialists in HIV clinics across Canada. The HIV status of infants is reported as "confirmed infected", "confirmed not infected", or "infection status not confirmed", according to the United States Centers for Disease Control and Prevention (CDC) surveillance case definitions for HIV infection (8). Tests for HIV deoxyribonucleic acid (DNA) or ribonucleic acid (RNA) (polymerase chain reaction) are used to confirm infection status by four months of age. Infants are classified as "not 
confirmed" if they have not yet received the required number of tests to determine their HIV status. The current status of confirmed infected infants is defined as "asymptomatic," "symptomatic," "died of AIDS," "died of a cause other than AIDS" or "lost to follow-up."

\section{Analysis}

We calculated the descriptive statistics from HASS and CPHSP for the years 2009 to 2014. The HASS data were stratified by: reporting province or territory, age group, sex, exposure category, and race/ethnicity. The CPHSP data presented are stratified by: year of infant birth, use of antiretroviral therapy (ART), infection status, race/ethnicity, and maternal country of birth.

The number and proportion of cases shown in this article reflect data received as part of routine HIV surveillance reporting; no statistical techniques were applied to account for missing data. Data in tables with small cell sizes $(n \leq 5)$ were reviewed for possible issues regarding data quality and case identification. Strategies such as data suppression or collapsing data categories were used if deemed necessary by national analysts or by provincial or territorial data providers.

\section{Results}

\section{HIV trends in Canada, over time}

The number of reported HIV cases has steadily decreased each year since 2009; there were 2,044 cases reported in 2014, down $1.7 \%$ from the 2,076 cases reported in 2013 and is the lowest annual number seen in the last two decades (Figure 1).

Figure 1: Number of reported HIV cases by year of test, Canada, 2009-2014

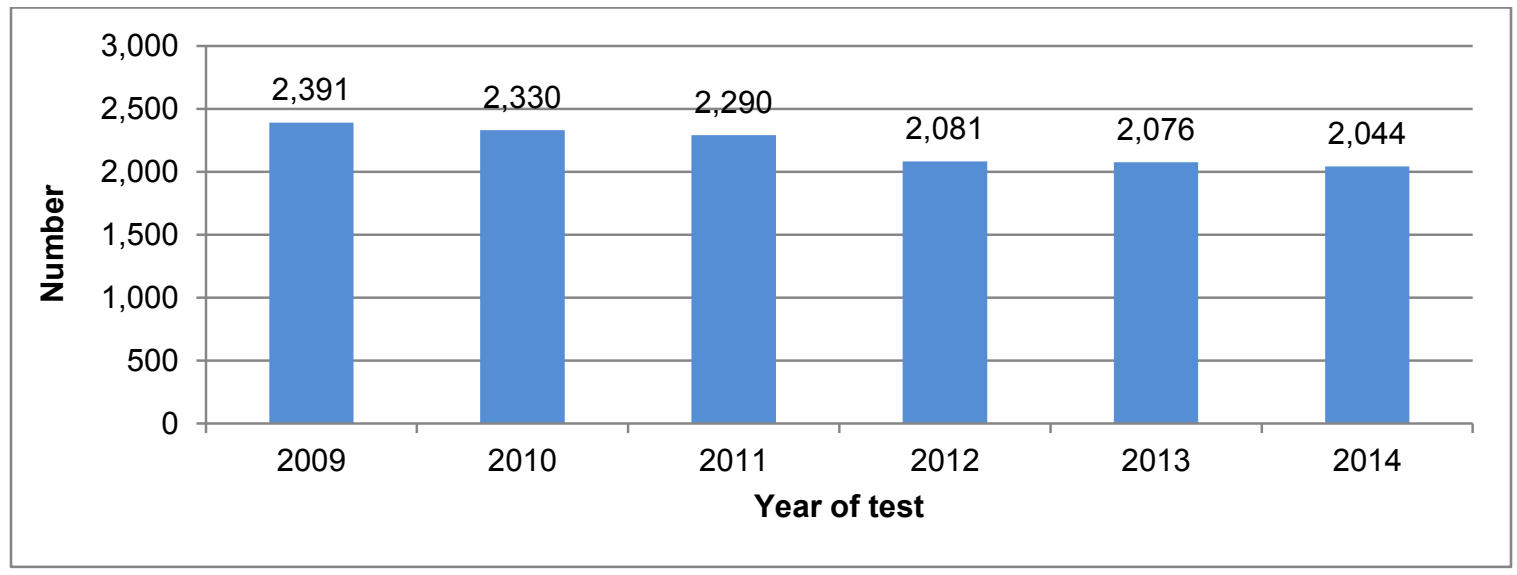

\section{Age and sex distribution}

The largest proportion of HIV cases are diagnosed among those aged 30 to 39 , accounting for approximately one third of cases each year, followed by those aged 40 to 49 . In recent years, the proportion of cases diagnosed at 50 years of age or over has increased; in 2014, this age group exceeded the proportion of reported cases of those aged 20 to 29 (Figure 2). It should be noted that these data reflect age at diagnosis, not necessarily age at infection. 
Figure 2: Percentage of reported HIV cases by age group and year of test, Canada, 2009-2014

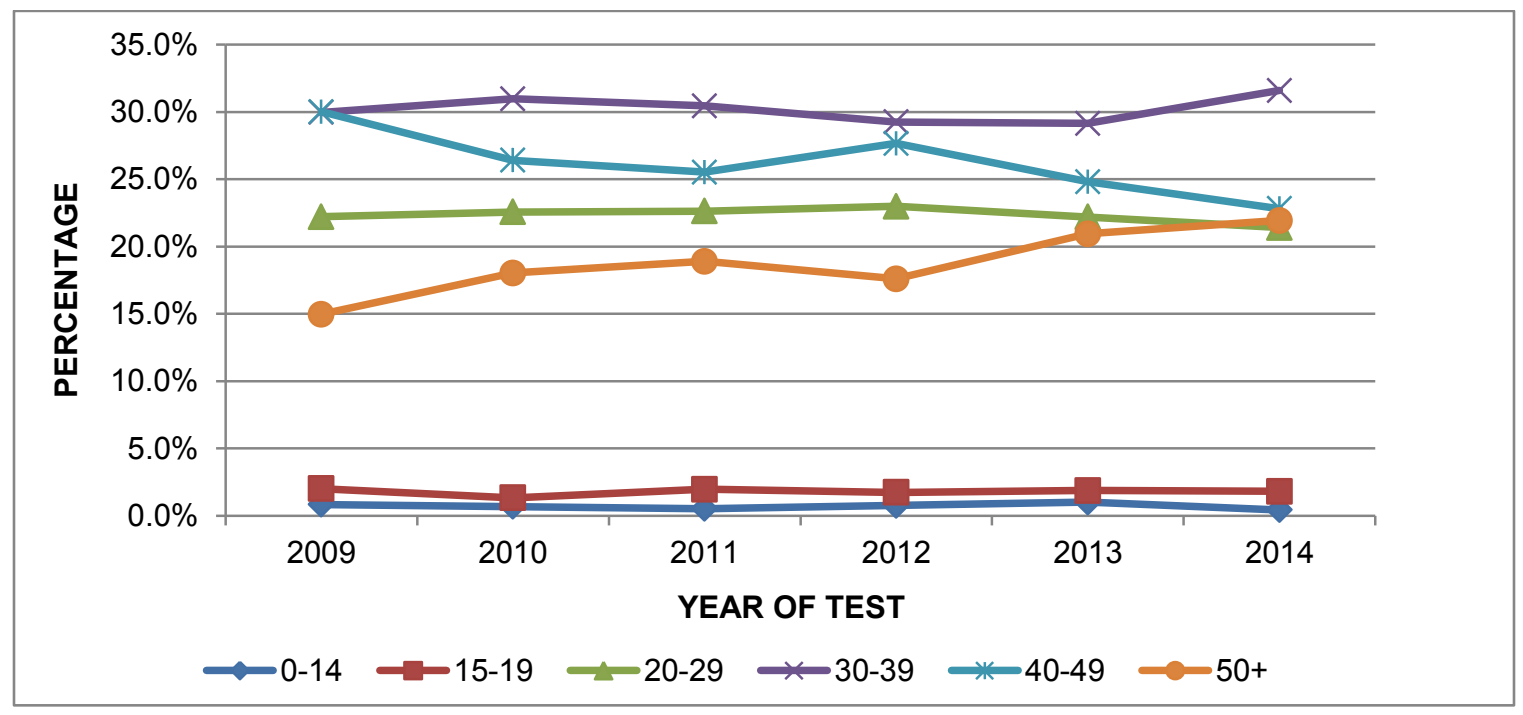

The majority of reported HIV cases have always been among males; in the 1990 s over $90 \%$ of annual HIV cases were male. From 2009 to 2014, the annual proportion of reported HIV cases that were female remained stable at approximately one quarter (range: $21.8 \%-25.8 \%$ ).

In general, there have been male-female differences in age at diagnosis-HIV was diagnosed at younger age groups ( $\leq 15,15-19$ and $20-29$ years of age) among females compared to males, where a greater proportion of HIV cases were diagnosed at older age groups (30-39, 40-49 and 50+ years of age).

\section{Exposure category}

Trends in exposure category have shifted since HIV reporting began. In the early stages of the epidemic, more than $80 \%$ of all reported HIV cases with a known exposure category were attributed to the "men who have sex with men" (MSM) exposure category. Although this exposure category is still predominant in Canada, the proportion has decreased over the years.

From 2009 to 2014, among cases where the exposure category was known (67.0\%), $45.9 \%$ of all reported HIV cases in adults ( $\geq 15$ years of age) were attributed to the MSM exposure category. The second most reported exposure category among adults was heterosexual contact (31.5\%), with a near even distribution of HIV cases attributed to heterosexual contact among people born in a country where HIV is endemic (Het-Endemic, 10.7\%), heterosexual contact with a person at risk (Het-Risk, 10.5\%), and heterosexual contact with no identified risk (Het-NIR, 10.3\%). The third most frequently reported exposure category among adults between 2009 and 2014 was injection drug use (IDU), accounting for $16.3 \%$ of reported HIV cases.

\section{Exposure category and sex}

The distribution of HIV cases among adult males and females ( $\geq 15$ years of age) differs with respect to exposure category. For 2009 to 2014, the MSM exposure category accounted for the greatest proportion $(60.2 \%)$ of reported HIV cases among adult males, whereas heterosexual contact (including the Het-Endemic, Het-Risk and Het-NIR groups) accounted for $66.2 \%$ of cases among adult females (Figure 3 and Figure 4). With respect to heterosexual contact, there was a substantial difference between males and females in the Het-Endemic exposure category, which accounted for $27.0 \%$ of heterosexual contact cases among females compared to $5.6 \%$ among males. 
The IDU exposure category accounted for approximately one quarter of adult female HIV cases (27.0\%), compared to up to $16.3 \%$ of adult male HIV cases (12.9\% via IDU exposure and up to $3.4 \%$ in the MSM/IDU category).

Figure 3: Proportion of reported HIV cases among adult males ( $\geq 15$ years of age) by exposure category, Canada, 2009-2014

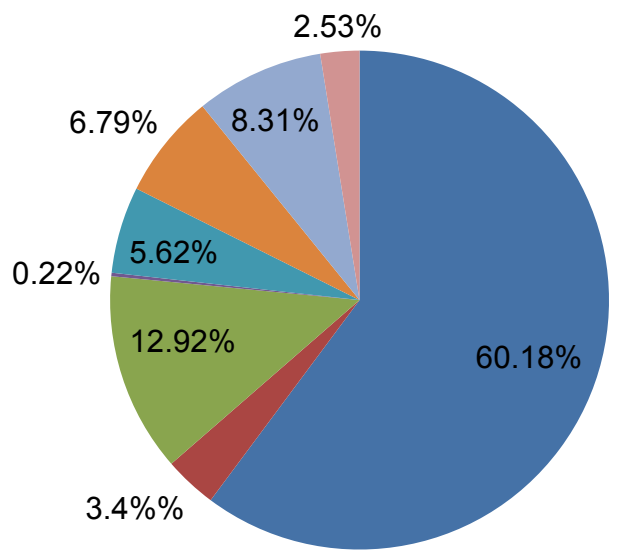

MSM $^{1}$
- MSM/IDU $^{2}$
IDU $^{3}$
Blood/Blood products
Het-Endemic
- Het-Risk
Het-NIR
- Other

${ }^{1} \mathrm{MSM}-$ men who have sex with men

${ }^{2} \mathrm{MSM} / \mathrm{IDU}$ - men who have sex with men and use injection drugs

${ }^{3} \mathrm{IDU}$ - injection drug use

${ }^{4}$ Het-Endemic - origin from an HIV-endemic country

${ }^{5}$ Het-Risk - sexual contact with a person at risk

${ }^{6} \mathrm{Het}-\mathrm{NIR}$ - heterosexual contact with no identified risk

Figure 4: Proportion of reported HIV cases among adult females ( $\geq 15$ years of age) by exposure category, Canada, 2009-2014

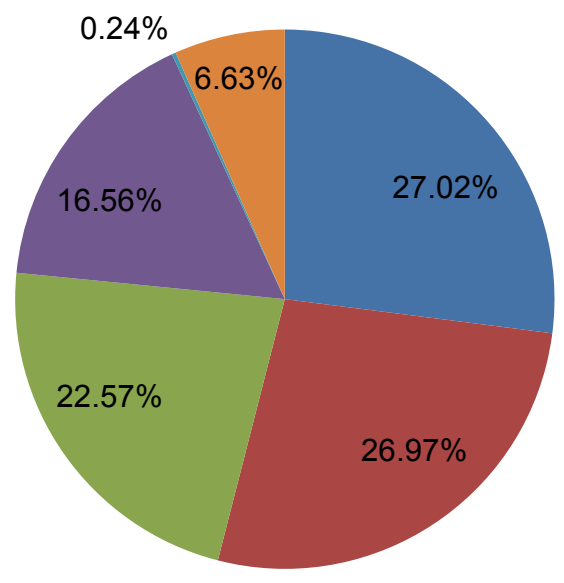

$\because$ IDU $^{1}$

- Het-Endemic ${ }^{2}$

- Het-Risk ${ }^{3}$

- Het-NIR ${ }^{4}$

Blood/Blood Products

- Other

IDU—injection drug use

${ }^{2}$ Het-Endemic - origin from an HIV-endemic country

${ }^{3}$ Het-Risk - sexual contact with a person at risk,

${ }^{4} \mathrm{Het}-\mathrm{NIR}$ - heterosexual contact with no identified risk 


\section{Race/ethnicity distribution}

For HIV cases reported from 2009 to 2014, information on race/ethnicity was available for nearly two thirds (59.8\%). While this is an improvement from the years prior to 2009, national trends presented in this article must be interpreted with caution given that a substantial number of HIV cases are not included in the race/ethnicity analysis. These data may not be fully representative of the national picture because race/ethnicity data for HIV cases from jurisdictions with more diverse populations are not included.

From 2009 to 2014, nearly one half of reported HIV cases were identified as White (46.8\%), followed by Aboriginal (19.2\%) and Black (18.1\%). The breakdown of the Aboriginal group is as follows:

$14.3 \%$ First Nations, 1.5\% Métis, 3.3\% Aboriginal-unspecified, and $0.0 \%$ Inuit.

The race/ethnicity distribution varies by sex: for 2009 to 2014, among males, the majority of cases were White (54.8\%), followed by Aboriginal (14.0\%) and Black (12.6\%) (Figure 5). By comparison, among females, there were equal proportions of Black (35.3\%) and Aboriginal (35.3\%), followed by White $(21.8 \%)$.

Figure 5: Proportion of reported HIV cases (all ages) by sex and race/ethnicity, Canada, 2009-2014

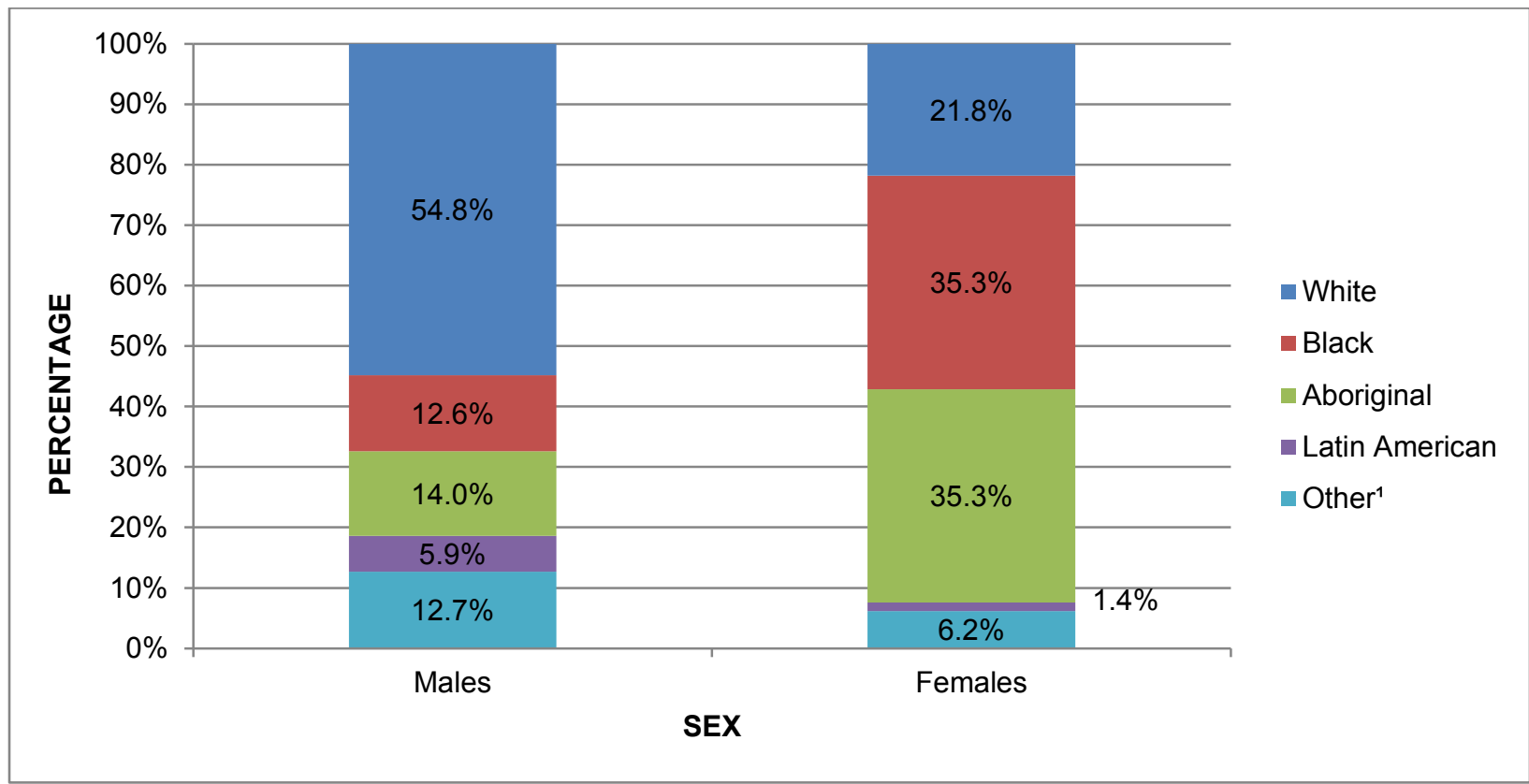

1“'Other" includes Asian, South Asian, West Asian, Arab and other race/ethnicity.

\section{Race/ethnicity and exposure category}

Information on both race/ethnicity and exposure category was available for $58.3 \%$ of reported cases from 2009 to 2014. Given that a substantial number of HIV cases were not reported with both of these data elements, this section is likely not representative of the overall distribution by exposure category and race/ethnicity in Canada.

Among HIV cases attributed to the MSM exposure category, the majority (65.8\%) were identified as White (Figure 6). Almost all HIV cases attributed to IDU exposure were either Aboriginal $(60.7 \%)$ or 
White (35.4\%). Among cases attributed to the Het-Endemic category (one of the exposure categories falling under heterosexual contact), $82.9 \%$ were identified as Black.

Figure 6: Proportion of reported HIV cases (all ages) by exposure category and race/ethnicity, Canada, 2009-2014

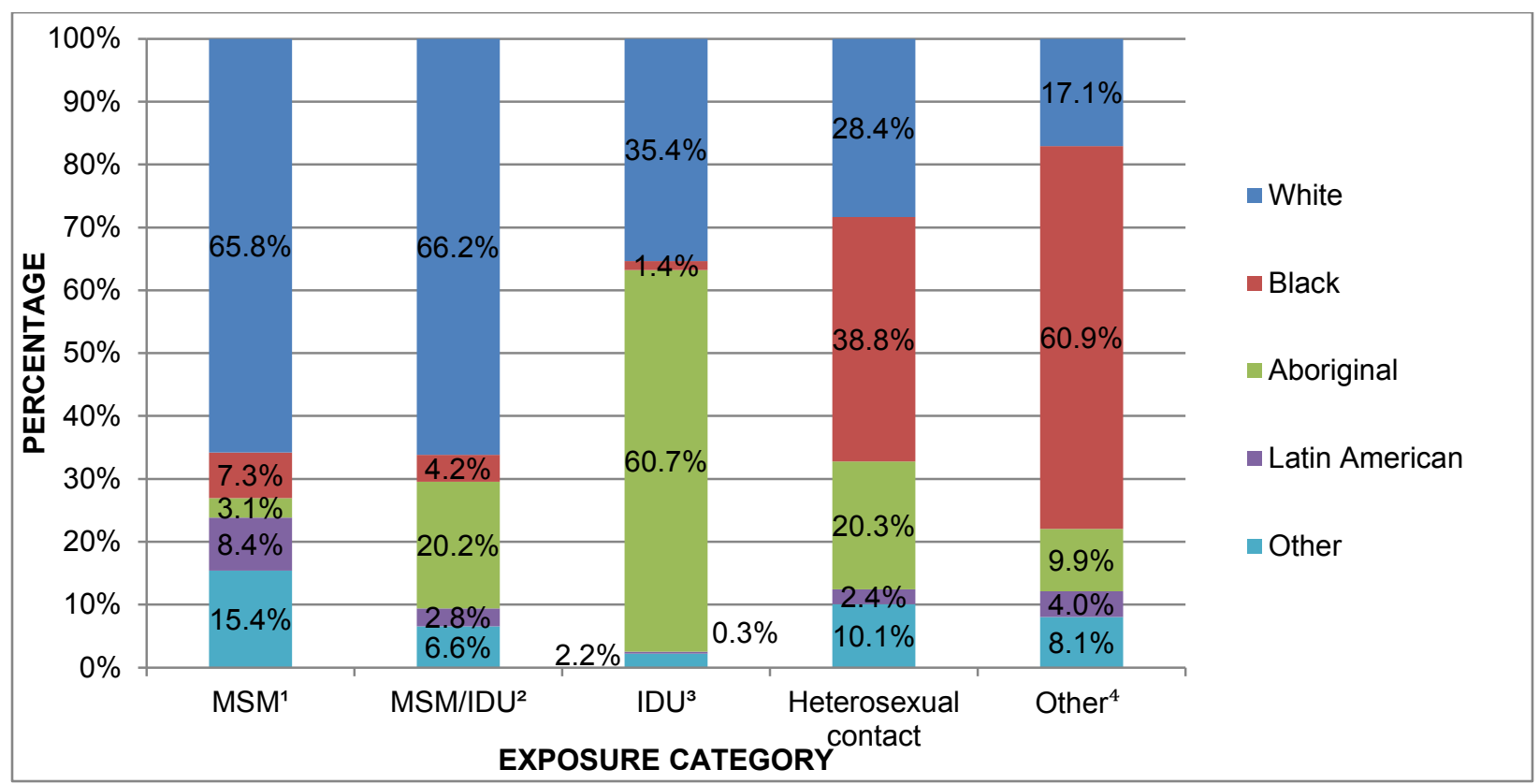

${ }^{1} \mathrm{MSM}-$ men who have sex with men

${ }^{2} \mathrm{MSM} / \mathrm{IDU}$ - men who have sex with men and use injection drugs

${ }^{3} \mathrm{IDU}$ - injection drug use

4"Other" includes Asian, South Asian, West Asian, Arab and other race/ethnicity.

\section{Geographic distribution}

The national rate (all ages) of HIV diagnosis in Canada in 2014 was 5.8 per 100,000 population. The highest rate (per 100,000 population) was in Saskatchewan (10.8), followed by Yukon (8.2), Alberta (6.7), Manitoba (6.6) and Ontario (6.1). In Saskatchewan, the all-age HIV diagnosis rate reached a high of 19.2 per 100,000 population in 2009 , with annual decreases thereafter. The remaining provinces and territories all reported HIV diagnosis rates below the national rate (ranging from 0.0 in Nunavut to 5.7 per 100,000 in British Columbia). Table 1 provides the number of reported cases and HIV diagnosis rate by province and territory for the years 2009 to 2014. 
Table 1: Number and HIV diagnosis rate (per 100,000 population), Canada and the provinces/territories, 2009-2014

\begin{tabular}{|c|c|c|c|c|c|c|c|c|c|c|c|c|c|c|c|}
\hline $\begin{array}{c}\text { Year of } \\
\text { diagnosis }\end{array}$ & $\begin{array}{c}\text { Numberl } \\
\text { Rate }\end{array}$ & Canada & ${ }^{1} \mathrm{BC}$ & ${ }^{2} \mathrm{YT}$ & ${ }^{3} \mathrm{AB}$ & ${ }^{4} \mathrm{NT}$ & ${ }^{5} \mathrm{NU}$ & ${ }^{6} \mathrm{SK}$ & ${ }^{7} \mathrm{MB}$ & ${ }^{8} \mathrm{ON}$ & ${ }^{9} Q C$ & ${ }^{10} \mathrm{NB}$ & ${ }^{11} \mathrm{NS}$ & ${ }^{12} \mathrm{PE}$ & ${ }^{13} \mathrm{NL}$ \\
\hline \multirow[t]{2}{*}{2009} & Number & 2,391 & 337 & $<5^{14}$ & 219 & $<5$ & 0 & 199 & 103 & 996 & 512 & $<5$ & 13 & 0 & 6 \\
\hline & Rate & 7.1 & 7.6 & -- & 6.0 & -- & 0.0 & 19.2 & 8.5 & 7.7 & 6.5 & -- & 1.4 & 0.0 & 1.2 \\
\hline \multirow[t]{2}{*}{2010} & Number & 2,330 & 300 & $<5$ & 204 & 0 & 0 & 174 & 121 & 1,025 & 476 & 8 & 15 & $<5$ & $<5$ \\
\hline & Rate & 6.9 & 6.7 & -- & 5.5 & 0.0 & 0.0 & 16.5 & 9.9 & 7.8 & 6.0 & 1.1 & 1.6 & -- & - \\
\hline \multirow[t]{2}{*}{2011} & Number & 2,290 & 288 & $<5$ & 224 & $<5$ & 0 & 188 & 77 & 1,003 & 477 & 10 & 15 & $<5$ & $<5$ \\
\hline & Rate & 6.7 & 6.4 & -- & 5.9 & -- & 0.0 & 17.6 & 6.2 & 7.6 & 6.0 & 1.3 & 1.6 & -- & - \\
\hline \multirow[t]{2}{*}{2012} & Number & 2,081 & 237 & $<5$ & 242 & $<5$ & 0 & 184 & 71 & 869 & 443 & $<5$ & 18 & $<5$ & 9 \\
\hline & Rate & 6.0 & 5.2 & -- & 6.2 & -- & 0.0 & 16.9 & 5.7 & 6.5 & 5.5 & -- & 1.9 & -- & 1.7 \\
\hline \multirow[t]{2}{*}{2013} & Number & 2,076 & 267 & $<5$ & 261 & $<5$ & 0 & 129 & 118 & 815 & 453 & 5 & 16 & $<5$ & 7 \\
\hline & Rate & 5.9 & 5.8 & -- & 6.5 & -- & 0.0 & 11.7 & 9.3 & 6.0 & 5.6 & 0.7 & 1.7 & -- & 1.3 \\
\hline \multirow[t]{2}{*}{2014} & Number & 2,044 & 262 & $<5$ & 276 & $<5$ & 0 & 121 & 85 & 837 & 435 & $<5$ & 10 & $<5$ & 10 \\
\hline & Rate & 5.8 & 5.7 & -- & 6.7 & -- & 0.0 & 10.8 & 6.6 & 6.1 & 5.3 & -- & 1.1 & -- & 1.9 \\
\hline
\end{tabular}

${ }^{1} \mathrm{BC}$ - British Columbia; ${ }^{2} \mathrm{YT}$-Yukon Territory; ${ }^{3} \mathrm{AB}$ - Alberta; ${ }^{4} \mathrm{NT}$ - Northwest Territories; ${ }^{5} \mathrm{NU}$-Nunavut; ${ }^{6} \mathrm{SK}$ - Saskatchewan;

${ }^{7} \mathrm{MB}$ - Manitoba; ${ }^{8} \mathrm{ON}$ — Ontario; ${ }^{9} \mathrm{QC}$ - Quebec; ${ }^{10} \mathrm{NB}$-New Brunswick; ${ }^{11} \mathrm{NS}$-Nova Scotia; ${ }^{12} \mathrm{PEI}$-Prince Edward Island;

${ }^{13} \mathrm{NL}-$ Newfoundland and Labrador; ${ }^{14}$ No rate is provided for numbers less than 5.

\section{Mother-to-child transmission (perinatal transmission)}

Each year between 2009 and 2014 the number of infants perinatally exposed to HIV in Canada fluctuated between 200 and 249 cases per year. In 2014, 233 infants were reported as being perinatally exposed, up $14.8 \%$ from the 207 reported in 2013 (Figure 7).

The proportion of HIV-positive mothers receiving antiretroviral therapy (ART) increased over time to 97.4\% in 2014 (Figure 7). Correspondingly, the number of infants born in Canada and confirmed to be HIV infected has decreased over the past five years. In 2009, there were eight confirmed HIV-infected cases $(4.0 \%)$ and in 2014 there were only two $(0.4 \%)$. 
Figure 7: Number of perinatally HIV-exposed infants and proportion of perinatally HIV exposed infants receiving perinatal antiretroviral therapy by year of birth, Canada, 2009-2014

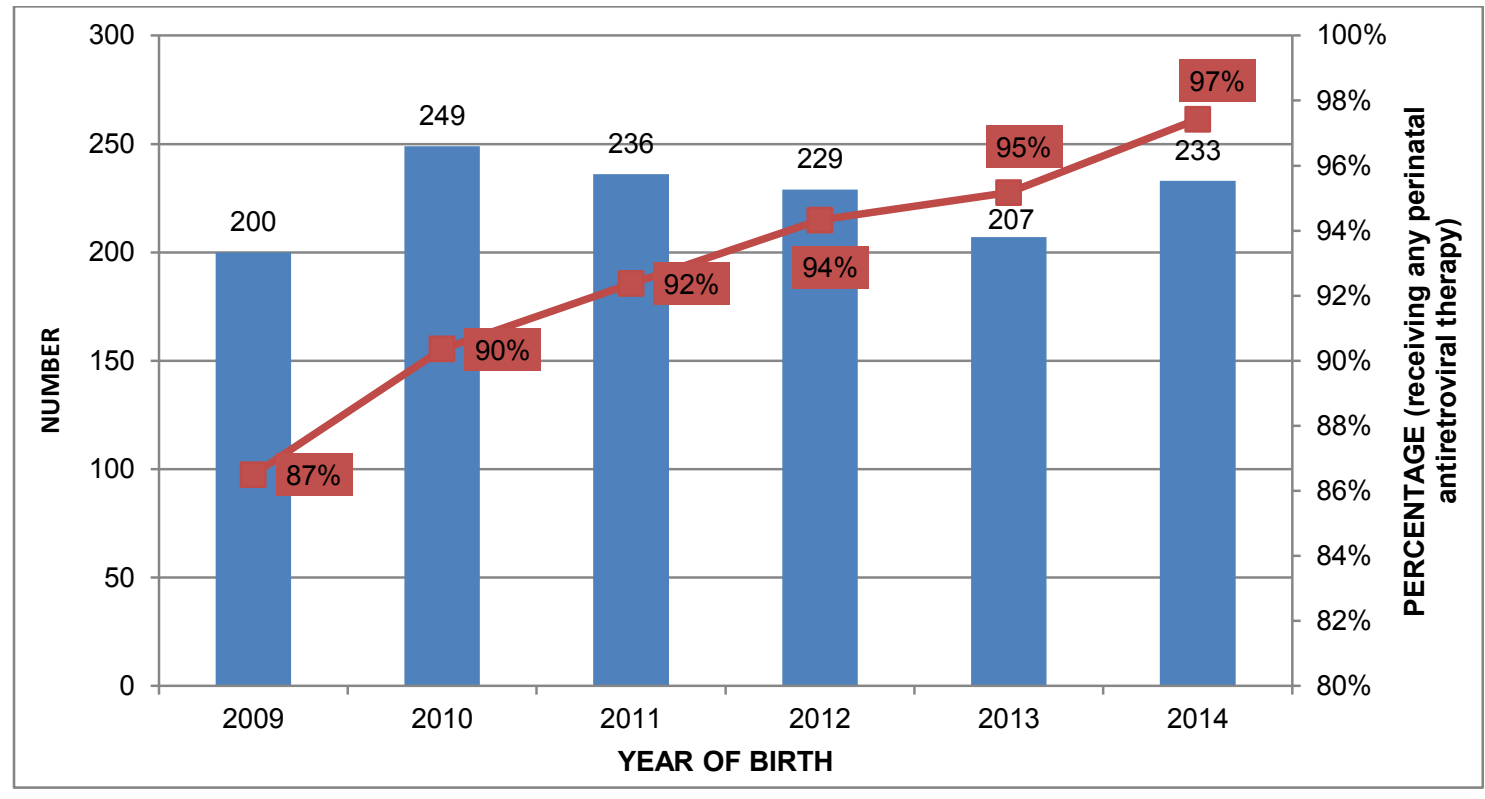

A review of all perinatally exposed infants from 2009 to 2014 shows that the majority $(77.4 \%)$ were born to mothers who acquired HIV infection through heterosexual contact, whereas nearly one fifth $(18.3 \%)$ were attributed to IDU exposure. With respect to race/ethnicity distribution of the infants, over half (54.1\%) were reported as Black, one fifth (19.6\%) as Aboriginal, and almost one fifth $(18.5 \%)$ as White. Maternal region of birth for the majority of infants was Africa (44.8\%), North America $(39.3 \%)$, or the Caribbean (6.6\%).

\section{Discussion}

The annual number of newly reported cases of HIV has been declining in Canada. In 2014, the number of new HIV cases reported to the Agency was the lowest reported in the last two decades; the rate is now 5.8 per 100,000 population. The MSM exposure category remains the predominant HIV exposure category in Canada overall, followed by heterosexual contact and IDU exposure, with some variation by sex, province/territory and race/ethnicity. There were distinct differences observed between males and females with respect to age at HIV diagnosis. HIV diagnosis tended to be at a younger age among females compared to males. Also, the proportion of HIV cases diagnosed among Canadians 50 years of age and older has increased gradually. Although the number of perinatally exposed infants increased slightly in 2014, the proportion of HIV-positive mothers receiving treatment has also increased and there were only two confirmed HIV-infected infants in Canada.

The national HIV surveillance system data have several limitations. There are variations in reporting practices across jurisdictions, reporting delays, under-reporting, duplicate reports, as well as missing or incomplete data. The completeness of epidemiologic information collected and submitted to the Agency varies by jurisdiction. In particular, exposure category and race/ethnicity information is incomplete for many case reports. Most significantly, Quebec does not submit exposure category or race/ethnicity information for HIV cases to the Agency; however, the province does publish provincial reports that include information on exposure category and race/ethnicity. In Ontario, completeness of exposure category and race/ethnicity data for HIV cases has changed over time. Completeness increased 
significantly for data from 2009 onwards due to the inclusion of supplementary data collected through the Ontario Laboratory Enhancement Program. Manitoba does not provide disaggregated data on Aboriginal populations. The perinatal surveillance data collected by CPHSP is sentinel-based; therefore, possible referral bias exists, as the main referral centres from each geographic area are used for data collection (7).

It is important to note that our data tend to understate the magnitude of the HIV epidemic. Surveillance data do not represent the total number of people infected with HIV (prevalence) or the number of people newly infected each year (incidence). Surveillance data can tell us only about people who have been diagnosed with HIV. Furthermore, because HIV is a chronic infection with a long latency period, many individuals who are newly infected in a given year may not receive a diagnosis until much later. As well there may be individual or societal behaviour changes over time that contribute to changes in observed disease trends, such as changes in HIV testing patterns (e.g., who comes forward for testing and when).

Awareness of HIV status remains a big issue. At the end of 2014 , an estimated $21 \%$ of the 75,500 individuals living with HIV in Canada were unaware of their infection. These people are hidden from the health care and disease monitoring systems, and thus cannot take advantage of appropriate prevention, care and treatment services until they are tested and diagnosed. Knowledge of HIV-positive status is also important to prevent vertical transmission.

In Canada, ongoing HIV transmission remains a concern. Since new HIV treatments have reduced HIV mortality and new infections continue to occur, the overall number of Canadians living with HIV infection will likely continue to increase in the years to come. So, although we have documented declining rates of new cases this does not mean the need for HIV care will decrease. And continued efforts are needed to reach the undiagnosed cases of HIV to decrease transmission (9).

Finally, given the variation in number of HIV case reports, as well as differences in the demographic profile of cases (i.e., race/ethnicity, age and sex) across provinces and territories, the data presented in this article highlights the need for population-specific interventions.

The national HIV surveillance data collected by the Agency continue to inform such work as: the estimates of HIV prevalence and incidence in Canada; the development and assessment of the HIV care cascade; the development and assessment of national public health guidance and recommendations (e.g., HIV testing guidelines); federal, provincial and territorial policy and program development to prevent and control HIV and AIDS; the development and dissemination of credible, evidence-based knowledge and public health guidance to support health professionals; and the development of targeted intervention strategies at local, provincial and national levels.

\section{Acknowledgements}

National-level HIV surveillance is possible as a result of all provinces and territories participating in, and setting directions for, HIV surveillance. Accordingly, the Agency acknowledges provincial and territorial HIVIAIDS coordinators, laboratories, health care providers and reporting physicians for providing the non-nominal confidential data that are used in this article.

The Agency also acknowledges the Canadian AIDS Pediatric Research Group for providing HIV data related to perinatal exposure.

\section{Conflict of interest}

None 


\section{References}

(1) Global summary of the AIDS epidemic, 2014.

http://www.who.int/hiv/data/epi_core_july2015.png?ua=1

(2) Joint United Nations Programme on AIDS (UNAIDS). 90-90-90-An ambitious treatment target to help end the AIDS epidemic. Geneva: UNAIDS; 2014.

http://www.unaids.org/sites/default/files/media_asset/90-90-90_en_0.pdf

(3) Public Health Agency of Canada. Summary: Estimates of HIV Incidence, Prevalence and Proportion Undiagnosed in Canada, 2014. Ottawa, ON: Minister of Public Works and Government Services Canada; 2015.

(4) Public Health Agency of Canada. HIV and AIDS in Canada: Surveillance Report to December 31, 2014. Ottawa, ON: Minister of Public Works and Government Services Canada; 2015.

(5) National Collaborating Centre for Infectious Diseases. Notifiable Diseases Database. http://nddb.ca/diseaseinfo/search/search disease\#search disease

(6) Public Health Agency of Canada. Case definitions for communicable diseases under national surveillance. CCDR. 2009 Nov;35S2:86-7.

(7) Forbes JC, Limenti AM, Singer J, Brophy JC, Bitnun A, Samson LM, et al.; Canadian Pediatric AIDS Research Group. A national review of vertical HIV transmission. AIDS. 2012 Mar 27;26(6):757-63. doi: 10.1097/QAD.0b013e328350995c.

(8) Schneider E, Whitmore S, Glynn MK, Dominguez K, Mitsch A, McKenna MT. Revised surveillance case definitions for HIV infection among adults, adolescents, and children aged $<18$ months and for HIV infection and AIDS among children aged 18 months to <13 years-United States, 2008. MMWR. 2008 Dec 5;57(RR10):1-16.

http://www.cdc.gov/mmwr/pdf/rr/rr5710.pdf

(9) Traversy GP, Austin T, Ha S, Timmerman K, Gale-Rowe M. An overview of recent evidence on barriers and facilitators to HIV testing. CCDR. 2015 Dec;41(12):304-321. 